\title{
"Wenn wir so weitermachen, fahren wir das System an die Wand»
}

\section{Matthias Scholer}

Wissenschaftsjournalist

\author{
Um die fortschreitende Kostenexplosion im Gesundheitswesen langfristig einzu- \\ dämmen, braucht es neue Lösungsansätze und Verzicht auf verschiedenen Ebenen. \\ Daniel Scheidegger, der neue Präsident der Schweizerischen Akademie der Medizi- \\ nischen Wissenschaften (SAMW), will sich dafür einsetzen, dass ein Kurswechsel \\ stattfindet - auch wenn nicht alle Player von den vorgeschlagenen Massnahmen \\ begeistert sein werden.
}

\begin{abstract}
Herr Scheidegger, Sie sind nun etwas mehr als 100 Tage Präsident der SAMW. Zeit für ein erstes Fazit. Daniel Scheidegger: Die Arbeit ist extrem spannend. Vieles ist für einen Quereinsteiger wie mich Neuland. Ich komme mir vor, wie jemand, der ein Paddelboot mietet und dieses auf ein fliessendes Gewässer setzt. Unter Beobachtung lässt man mich sogar schon mal steuern. Ansonsten lass ich aber auch ganz gerne paddeln, da ich noch nicht genau weiss, wo welche Untiefen auf uns warten. Mein Vorgänger und der Vorstand haben in den letzten Jahren vieles aufgegleist, das erst jetzt zum Tragen kommt. Dabei gibt es auch Themen, bei denen ich noch viel lernen kann.
\end{abstract}

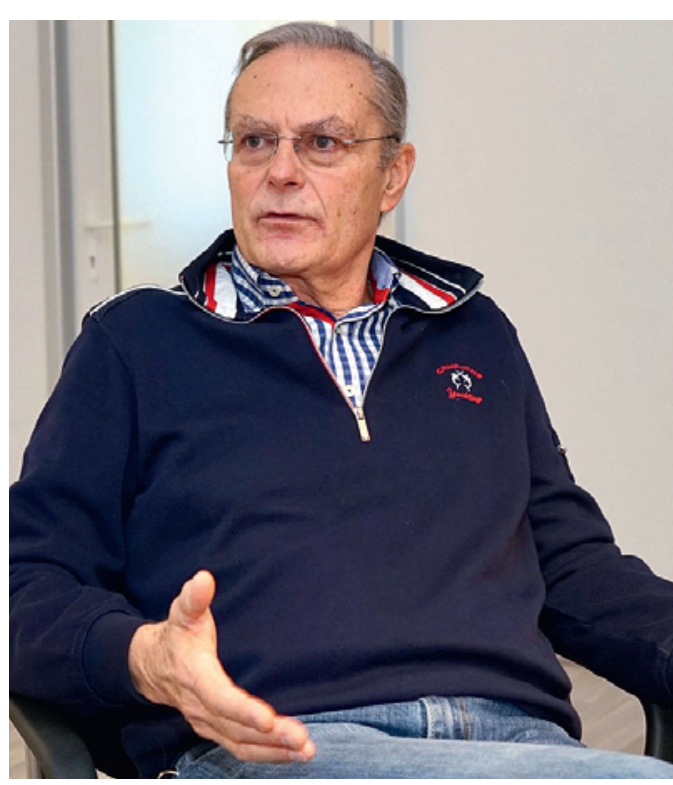

\section{Zur Person}

Daniel Scheidegger erlangte nach seiner Ausbildung die Facharzttitel Innere Medizin, Anästhesiologie und Reanimation und Intensivmedizin. Nach seiner Habilitation durchlief Scheidegger verschiedene leitende Positionen im Departement Anästhesie des Kantonsspitals Basel. Er war zudem federführend beim Aufbau des anonymen Fehlermeldesystems CIRS an Schweizer Spitälern. Daniel Scheidegger war bis am 28. Februar Präsident des Fachorgans Hochspezialisierte Medizin der Gesundheitsdirektorenkonferenz und seit 2005 Mitglied des Senats der SAMW.

\section{Können Sie uns ein Beispiel machen?}

Dazu gehört unter anderem die Personalisierte Medizin. Mein Vorgänger Peter Meier-Abt begann schon früh in seiner Amtszeit, die Rahmenbedingungen zu schaffen, damit die Schweiz den Anschluss bei der Nutzung gesundheitsbezogener Daten in der Forschung nicht verpasst. Mit Erfolg. Der SAMW gelang es, eine entsprechende, nationale Plattform zu schaffen. Im Verbund mit den ETHs, Universitäten und Universitätsspitälern wurde das «Swiss Personalized Health Network» gegründet. Das Staatssekretariat für Bildung, Forschung und Innovation hat diese Initiative angenommen und plant, die für die Umsetzung nötigen finanziellen Mittel zur Verfügung zu stellen. Die SAMW wird dieses Netzwerk operativ leiten. Dies ist für mich ein ziemlich neues Thema, das ich sehr spannend finde, und ich arbeite mich gerne in diese Materie ein.

\section{Welches sind die Themen, bei denen Sie sich bereits jetzt «zu Hause fühlen»?}

Unsere Akademie befasst sich seit längerem mit Lösungsansätzen, um einerseits die Kostenexplosion im Gesundheitswesen eindämmen und andererseits den Personalnotstand in der Grundversorgung entschärfen zu können. Dafür geeignete Massnahmen und Ideen werden bei der SAMW unter dem Begriff «Nachhaltige Medizin» bewirtschaftet. Da ich mich schon seit vielen Jahren intensiv mit Fragen rund um die Patientensicherheit und Qualität befasse und aus eigener beruflicher Erfahrung die Auswirkung von medizinischen Entwicklungen und Trends auf unser Gesundheitswesen kenne, sind dies auch Themen, die ich als Präsident vertieft angehen möchte. 
Ist der "Patient Gesundheitswesen" noch zu retten?

Wenn wir so weitermachen wie bisher, fahren wir das System an die Wand. Während der letzten zehn Jahre pumpten wir immer mehr Geld in unser Gesundheitssystem, ohne jedoch um denselben Faktor besser zu werden. Aber wen wundert's? Wir setzen immer mehr neue, meist sehr teure Technologien oder Methoden ein, bei denen meist noch nicht einmal erwiesen ist, ob sie überhaupt einen Mehrwert bringen. Solche Innovationen sollten zuerst an ausgewählten Spitälern getestet und die dabei gewonnenen Daten in auditierten Registern gesammelt werden. Ähnlich verhält es sich mit den Ausgaben bei der Infrastruktur. Die Planungssumme für Spitalumbauten ist höher als die veranschlagten Kosten der NEAT. Wenn Sie heute wissen wollen, wo in einer Gemeinde das Spital ist, müssen Sie bloss nach Baukränen Ausschau halten. Jede Klinik möchte das Angebot erweitern und muss dafür die Infrastruktur ausbauen. Obwohl es äusserst fraglich ist, ob ein Spital künftig die Kosten durch Mehroperationen und stationäre Behandlungen decken kann. So kann es nicht weitergehen.

\section{Wie kann man diesem Bauboom bei den Spitälern entgegenwirken?}

Was wir langfristig brauchen, sind grosse, kantonsgrenzenübergreifende Gesundheitsregionen, in denen nicht jedes Spital alle Leistungen anbietet. Ein Thema, das zum Glück auch in der Strategie "Gesundheit 2020" aufgenommen wurde.

\section{Bislang waren die Versuche, solche Netzwerke zu etablieren, nicht sehr erfolgreich.}

Das stimmt. Aber sie hatten alle denselben Systemfehler: Es fehlte eine gemeinsame Trägerschaft. Eine kantonsgrenzenüberschreitende Gesundheitsregion funktioniert nur, wenn man eine gemeinsame Kasse führt. Sonst kommt verständlicherweise Futterneid auf. Man muss auch darauf achten, innerhalb einer Region die Cashcows und die prestigeträchtigen Disziplinen topographisch sinnvoll zu verteilen und die bisherigen Ausrichtungen der involvierten Kliniken zu respektieren. Ansonsten gehen die Emotionen hoch und zerstören gleich zu Beginn alle guten Ideen.

\section{In den beiden Basel werden die Pläne für eine gemeinsame Gesundheitsversorgung immer konkreter.}

Da könnte sich tatsächlich eine gute Möglichkeit ergeben, mit neuen Modellen zu experimentieren, ohne dass gleich alles in Stein gemeisselt wird. Ganz wichtig ist es jedoch, dass eine Gesundheitsregion von einer externen Begleitforschung unterstützt wird. Nur so lässt sich eine solide Datengrundlage schaffen, auf die man sich einerseits bei politischen Diskussionen stützen kann und andererseits Schwachstellen frühzeitig erkennt.

«Vieles ist für einen Quereinsteiger wie mich Neuland.»

Wenn man über die stetig steigenden Gesundheitskosten spricht, werden immer wieder die hohen Tarife der Spezialisten ins Spiel gebracht. Muss man nicht auch in diesem Bereich eingreifen?

Die aktuellen TARMED-Verhandlungen gehen ja in diese Richtung. Und das ist gut so. Es gibt heute tatsächlich groteske Lohndiskrepanzen. Wenn man beispielsweise sieht, was ein Radiologe verrechnen kann, wenn er einige Bilder anschaut, ohne auch nur einmal mit den Patienten zu sprechen, und diese Einkünfte mit denen eines Hausarztes vergleicht, der im Viertelstundentakt Patientengespräche führt und schwierige Entscheidungen fällt, dann stimmt etwas nicht. Ich mag mich noch an meine Assistenzzeit erinnern, als der "Blick» eine Titelgeschichte über einen Arzt brachte, der eine Million im Jahr verdiente. Dazumal war dies ein Skandal. Heute wäre das keine Schlagzeile mehr wert. Seit den Lohnexzessen von Managern wie Ospel und Vasella sind die Zahlen völlig aus dem Gleichgewicht geraten. Doch diese Bankermentalität gehört einfach nicht in die Medizin.

\section{Aber Sie haben doch als Chefarzt wohl auch nicht gerade schlecht verdient?}

Ich war festangestellt und hatte einen fixen, vernünftigen Lohn. Vernünftig bedeutet in diesem Zusammenhang für mich, dass ich keine schlaflosen Nächte haben musste, wenn eine Reparatur am Auto anstand oder wir uns Ferien leisten wollten. Auch ich war und bin der Auffassung, dass Ärzte einen Lohn haben sollten, der unseren langen Arbeitszeiten und Verantwortung entspricht. Das Grundübel in diesem Bereich sind die falsch gesetzten Anreize. Je kränker meine Patienten, desto höher mein Verdienst. Und: Ich verdiene mehr, wenn ich etwas an einem Patienten mache, als wenn ich ihm versuche zu erklären, dass es besser wäre zuzuwarten. Das ist im Grund doch pervers. Solange die Anreize so gesetzt sind, solange haben wir in unserem System ein massives Problem.

\section{Aber die Motivation für ein Medizinstudium ist doch,} Kranken zu helfen.

Das ist unbestritten die beste Grundmotivation für ein Studium. Aber dass man später in der Laufbahn immer mehr Kranke will, um mehr zu verdienen, ist der Feh- 
ler. Der Ansatz, man sollte als Arzt für die Gesundheit seiner Population bezahlt werden, hat für mich etwas Faszinierendes. Dabei dürfen wir aber nicht vergessen, dass es viel mehr Zeit benötigt, einem Patienten zu erklären, weshalb er eine Behandlung nicht braucht, als eine Therapie anzuordnen. Zudem ist die Gefahr gross, dass er auf dem Nachhauseweg zur Konkurrenz geht, weil er schlicht eine Behandlung erwartet.

\section{«Diese Bankermentalität gehört einfach nicht in die Medizin.»}

\section{Also sind auch die Patienten mit ihrer Erwartungs- haltung nicht ganz unschuldig, dass sich ein Arzt verpflichtet fühlt, "etwas zu machen»?}

Die Patienten müssen für eine Abwägung nicht nur umfassend über ihr Gesundheitsproblem und dessen Therapiemöglichkeiten, sondern auch über mögliche negative Verläufe nach einer Behandlung informiert werden. Doch in den Medien wird diese Seite praktisch immer ausgeblendet. In den Gesundheitssendungen, die bald auf jedem Fernsehkanal angeboten werden, hören wir immer nur das Gute. Niemand spricht über Komplikationen. Da sieht man nur wie ein C-Promi nach einer Knieoperation wieder Golf spielt. Aber die Patientengeschichten, bei denen es beispielsweise nach einem Eingriff zu einer Gelenksinfektion kommt, werden in diesen Formaten nie erzählt. Doch auch sie sind ein Teil des Gesamtbildes. Und bloss weil jemand nicht mehr wie in jungen Jahren acht Stunden Skifahren kann, braucht es weder ein MRI noch eine Knie-OP.

\section{Aber solange es die Versicherung bezahlt, wird sich diese Haltung doch kaum ändern.}

Ich denke, wir müssen den Versicherungsnehmern viel klarer machen, dass wir ein solidarisch getragenes System haben. Das Geld, das ein Prämienzahler jeden Monat zahlt, ist kein Kredit, auf den man im Krankheitsfall zurückgreifen kann. Es muss aufgezeigt werden, dass mein Geld, das ich einzahle, hier und heute an Patienten geht, die eine Gesundheitsleistung benötigen. So finanzieren wir ein vorbildliches System, dank dem jedermann ansonsten unbezahlbare Leistungen beziehen kann. Für diese Fälle braucht es Krankenkassen. Aber dass man für jeden Hustensirup oder Kopfschmerzmittel zusätzlich die Versicherung belastet, ist meines Erachtens falsch.

Mögliche Strukturanpassungen rund um den Begriff "Nachhaltige Medizin" hat die SAMW in einem Positionspapier zusammengefasst, das auch in der $S A ̈ Z^{1}$ veröffentlicht wurde. Die Stellungnahme der

\section{FMH dazu zeigte, dass die Vorstellungen über} wirksame Massnahmen weit auseinandergehen.

Wir müssen nicht immer gleicher Meinung mit der Standesorganisation sein. Die Akademie versteht sich nicht als Vertreterin der Ärzteschaft, auch wenn mit Jürg Schlup der Präsident der FMH im Vorstand sitzt. Die Aufgabe der SAMW ist es vielmehr, das ganze Spektrum auszuleuchten. Deshalb setzt sich unser Vorstand aus Vertretern verschiedener Disziplinen inklusive den Pflegewissenschaften und der Forschung zusammen. Jeder soll die Möglichkeit haben, sich frei und kritisch zu äussern. Ich finde es toll, dass sich die Schweiz einen solchen Thinktank leistet.

\section{In einem Interview mit dem «Tages-Anzeiger» haben} Sie gesagt, Ärzte verhalten sich wie Messies. Haben Sie böse Reaktionen erhalten?

Das habe ich zwar erwartet, aber es kam ganz anders. Von den über 90 Mails, die ich nach dem Interview erhalten habe, war nur ein einziges negativ. Das zeigt auch, dass die Zeit reifer wurde und Themen, die bislang nur hinter verschlossenen Türen diskutiert wurden, viele Ärzte in ihrem Berufsalltag beschäftigen. Insbesondere Mediziner im Spital leiden häufig unter dem Druck der Ökonomen in den Spitalleitungen.

"Auch wir Ärzte müssen lernen, Verzicht zu üben.»

Aber, und deshalb habe ich auch den Begriff «Messie» gebraucht, müssen auch wir Ärzte lernen, Verzicht zu üben. Wir müssen lernen, dass, wenn man neue Behandlungstechniken übernimmt, alte Verfahren obsolet werden können. So ersetzt in vielen Fällen ein MRI beispielsweise die herkömmlichen Röntgenaufnahmen. Es macht fast nie Sinn, zwei Systeme parallel weiterzuführen.

\section{Verzicht auf vielen Ebenen, Versorgungsstrukturen, die unser föderalistisch geprägtes Denkmuster durchbrechen, und neue Anreizsysteme. Ist eine Umsetzung dieser Riesenschritte in absehbarer Zukunft überhaupt realistisch?}

Ich bin nicht naiv, und mir ist bewusst, dass solch grosse Vorhaben nur mit kleinen Schritten nachhaltig zu erreichen sind. Es ist nun jedoch höchste Zeit, die Situation ernst zu nehmen und aktiv an einer Lösung zu arbeiten. Denn wenn der Bund einschreiten muss, wird es uns alle viel mehr schmerzen. Aber ich habe die Hoffnung noch nicht aufgegeben, dass wir gemeinsam das Ruder herumreissen können.

Bildnachweis Matthias Scholer 